\title{
The effects of axotomy on the conduction of action potentials in peripheral sensory and motor nerve fibres
}

\author{
T E M IL NER AND R B STEIN \\ From the Department of Physiology, University of Alberta, Edmonton, Canada
}

SUMMARY Medial gastrocnemius and sural nerves in one hindlimb of the cat were transected and prevented from regenerating. After periods ranging from 29-273 days, compound action potentials were recorded from axotomised and contralateral control nerves. The amplitude and integrated area of action potentials decreased and conduction velocity slowed following axotomy. The area under compound action potentials generated by stimulating sensory fibres declined significantly faster than that generated by stimulating motor fibres. Analysis of changes in whole nerve conduction velocity distributions showed that the velocities of fast conducting sensory fibres decreased at the most rapid rate. The conduction velocities of motor fibres and slow sensory fibres declined at significantly slower rates. The loss of electrical activity in the largest sensory nerve fibres following axotomy, may play a role in determining the faster rate at which their action potentials deteriorate.

The process of peripheral nerve atrophy following axotomy has been extensively studied in terms of its morphological consequences (reviewed by Sunderland ${ }^{1}$ ). However, less is known about the electrophysiological changes which affect the ability of axons to conduct impulses. Conduction velocity falls as fibre diameter decreases; ${ }^{23}$ the two recover only if regenerating axon sprouts establish functional connections with their end organs. ${ }^{45}$ Prevention of functional recovery does not necessarily lead to complete loss of axonal function. Spontaneous and evoked neural activity can be recorded long after a nerve has been severed. ${ }^{6-8}$ Although the magnitude of the activity declines for some months following section of the nerve, it may eventually approach a steady-state level. ${ }^{8}$

Do all axons in a severed nerve degenerate to the same extent or are some fibres particularly affected? Indications that sensory fibres are more severely affected than motor fibres ${ }^{89^{-11}}$ have been confirmed recently by Hoffer et al. ${ }^{12}$ Compound action potentials were recorded from dorsal and

Address for reprint requests: TE Milner, Department of Physiology University of Alberta, Edmonton, Alberta, Canada T6G 2H7.

Accepted 21 January 1981 ventral roots following stimulation of normal and previously severed sensory and muscle nerves. The integrated area of dorsal root compound action potentials was found to decrease more rapidly than that of ventral root compound action potentials over a period of approximately 250 days. Although distinguishing between sensory and motor axons, this type of analysis did not provide the detail necessary to resolve possible differential effects on axons classified according to size or conduction velocity.

We decided to use conduction velocity distributions computed from compound action potentials as a tool for examining differential effects of axotomy on various classes of nerve fibres. The details of the method used in computing the distributions is outlined in the preceding paper. ${ }^{13}$ From the conduction velocity distributions the viability of different classes of axons could be determined at various times following axotomy.

\section{Methods}

Data were obtained from experiments conducted on 26 adult cats of both sexes, nine of which were normal animals and 17 in which the sural and medial gastrocnemius (MG) nerves of the left hindlimb had been 
surgically sectioned some time prior to the experiment. Transection of the nerves was performed under aseptic conditions. The animals were initially anaesthetised with Nembutal and maintained on Halothane for the duration of surgery. The nerves were ligated proximal and distal to the point of section and then cut cleanly with scissors. The MG nerve was severed near its point of entry into the MG muscle and the sural nerve distal to the midpoint of its course over the lateral gastrocnemius muscle. The proximal stump of the severed nerve was sutured to a Silastic sheet approximately $1 \mathrm{~cm}$ square. Care was taken to prevent reinnervation by reflecting the MG nerve away from its muscle and suturing the Silastic sheet to more proximal muscles. In the case of the sural nerve the sheet was turned over onto the nerve and sutured to the lateral gastrocnemius muscle. In some of the earliest attempts the precautionary procedure was not as thorough and reinnervation did occur.

Acute experiments under deep Nembutal anaesthesia were conducted after periods ranging from 29 273 days following initial surgery. Nerves and spinal roots of both hindlimbs were prepared for stimulation and recording. The right hindlimb served as a control. Spinal roots from the L6 to S2 levels were exposed bilaterally by laminectomy. Extensive denervation of the hindlimbs was required to minimise artefact due to muscle activity when stimulating ventral roots. All branches of the sciatic nerve with the exception of the two nerves of interest were cut. The MG and sural nerves were then dissected free from surrounding tissue over a length of 15-25 mm. The cat was mounted in a stereotaxic frame with both hindlimbs extended and securely clamped at the knee and ankle. Paraffin oil pools fashioned from skin flaps bathed the spinal cord and the hindlimb nerves. Their temperature was maintained at $36^{\circ} \pm 2^{\circ}$ by radiant heat. Body temperature was kept in the same range by means of a thermostatically controlled heating pad. Just prior to recording, nerves were ligated and cut distal to the ligature. Axotomised MG nerves were cut $5-10 \mathrm{~mm}$ proximal to the neuroma while the longer sural nerves were cut farther from the neuroma.

Recording and sampling procedures have been described in some detail in the preceding paper. ${ }^{13}$ Compound potentials from control nerves were sampled at a $20 \mathrm{kHz}$ rate. Lower sampling rates (down to $6 \mathrm{kHz}$ ) were used for compound potentials recorded from axotomised nerves because of their longer duration due to slower conducting fibres. Generally, the longer the time which had elapsed since axotomy the lower the sampling rate. In addition to recording compound action potentials from the nerves, the nerves were stimulated supramaximally in order to record compound potentials from the spinal roots.

\section{Results}

Figure 1 compares the mean conduction velocity distributions obtained from control and contra- lateral axotomised sural nerves of seven cats in experiments conducted 29-71 days following axotomy. The conduction velocity distributions were computed as described in the preceding pasper ${ }^{13}$ and have been plotted here in three forms.

The first plot (fig 1A) is a normalised histogram on a linear scale of conduction velocity with bins of equal width. The conduction distance, the latency to onset of the compound action potential ard the sampling rate determine the bin width. Because of the inverse relationship between conduction velocity and time, conduction velocity insrements corresponding to the sampling intervals of the digitised compound action potential decrease as the conduction time increases. Using histogram bins of equal width fails to take advantage of the higher resolution at lower conduction velocities offered by this relationship.

In fig $1 \mathrm{~B}$ the bin width is allowed to decrease as conduction velocity decreases providing a consequent increase in the resolution of the percentage of slowly conducting fibres. This was accomplished by a threefold increase in the number of histogram bins. Note that the histograms have been plotted on a logarithmic scale of conduction velocity and hence the bin widths appear approximately equal. The choice of a logarithmic scale was prompted by the need for a simple method of qualitatively comparing the relative effects of axotomy on fast and slowly conducting fibres, as explained below.

Cumulative histograms (fig 1C) proved useful in comparing the conduction velocity distributions of control and axotomised nerves. For any given condustion velocity the cumulative histogram represerits the percentage of fibres in the distribution which conduct at velocities less than or equal to the.t velocity. By using a logarithmic scale of conduction velocity it is possible to determine the relative effects of axotomy on the fast and slowly conducting populations of fibres simply by examining the shifts in the conduction velocity distribution. A parallel shift of the distribution to the lefi: without a change in its shape would imply that both fast and slowly conducting populations had slowed by the same relative amount. Differential effects would be apparent if the shift was not parallel.

The sural nerve conduction velocity distributions in fig 1 show this quite clearly. From the left hand side of fig $1 \mathrm{~A}$ and $1 \mathrm{~B}$ it is evident that the control conduction velocity distribution is bimodal. The same information is conveyed by the plateau in the cumulative distribution of fig 1C which, in addition, clearly shows that approximately $40 \%$ 

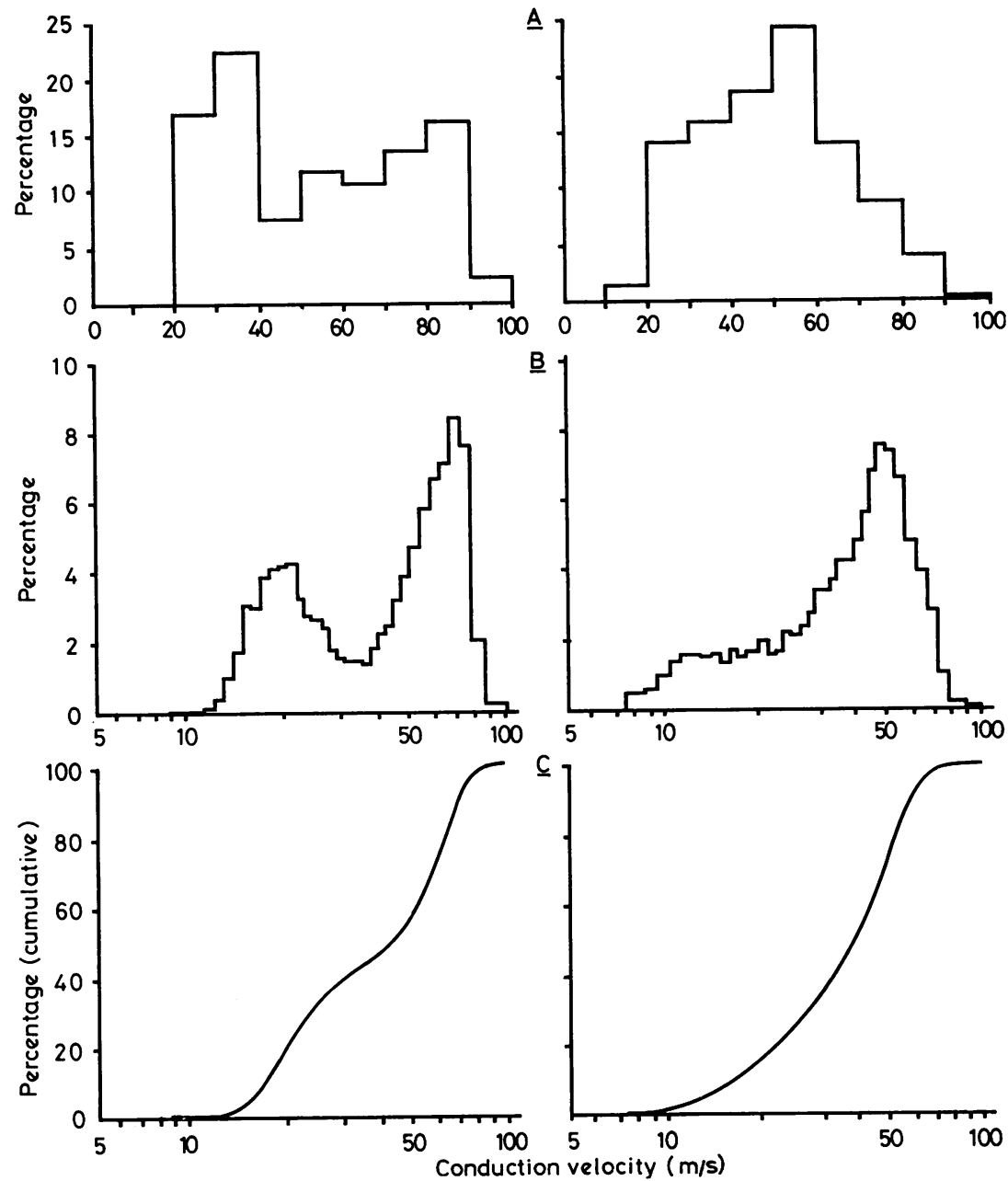

Fig 1 The mean conduction velocity distributions for control (left $)$ and axotomised (right) sural nerves 29-71 days following axotomy plotted as: $A$ conventional histograms on a linear scale of conduction velocity; $B$ conventional histograms on a logarithmic scale of conduction velocity; $C$ cumulative distributions on $a$ logarithmic scale of conduction velocity. Refer to text for explanation.

of the fibres were slowly conducting fibres and $60 \%$ were fast conducting. Within the first two months following axotomy (right hand side of fig 1) the bimodal nature of the distribution was lost and the plateau disappeared from the cumulative distribution.

The loss of the plateau could have been due to a greater slowing of fast conducting fibres (filling the gap between the two populations), a relative loss of slowly conducting fibres or a combination of the two. By superimposing the cumulative distributions of the control and axotomised nerves (fig 2 (top)) one can see that there was a clear decrease in the conduction velocity of the fastest conducting fibres (the leftward arrow indicates that the $80 \%$ point has shifted from about $60 \mathrm{~m} / \mathrm{s}$ to $50 \mathrm{~m} / \mathrm{s}$ ). However, the two distributions actually cross over which suggests that whereas initially $40 \%$ of the fibres were slowly conducting, the relative number has decreased to about $20 \%$ (diagonal arrow).

The differences may have been exaggerated by a tendency for the single unit potentials recorded from slowly conducting fibres to be diphasic even under monophasic recording conditions. ${ }^{14}$ Slowly conducting fibres sometimes had a substantial 


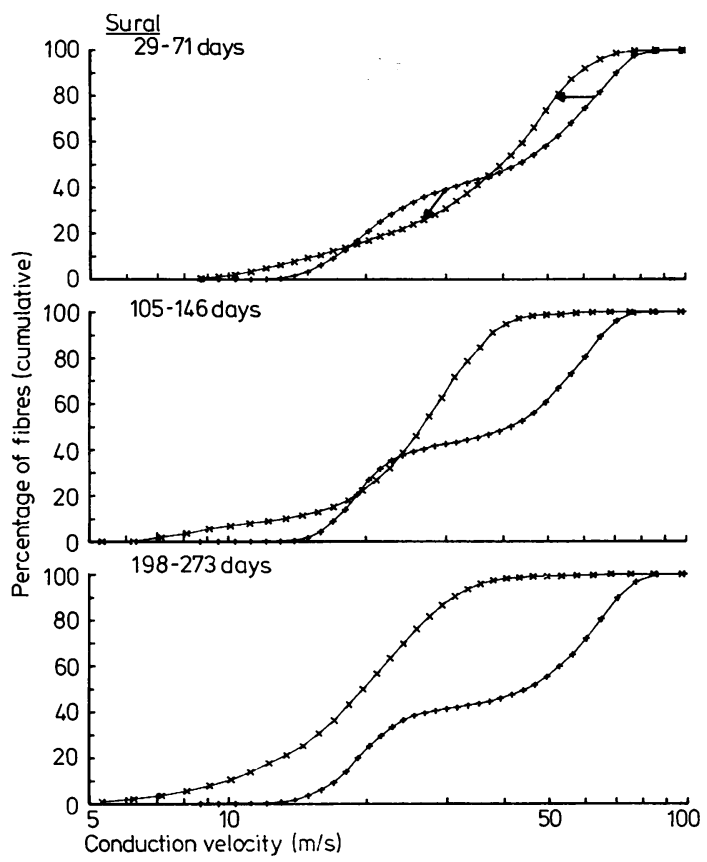

Fig 2 Progressive changes in the conduction velocity distribution of sural nerves following axotomy. Conduction velocity distributions for axotomised nerves $(X)$ examined in the indicated time periods were averaged and superimposed on the mean conduction velocity distributions of the corresponding control nerves $(+)$. Refer to text for discussion.

negative phase while fast conducting fibres showed relatively less negativity. As a result, there may have been some cancellation of positive and negative phases among slowly conducting fibres of slightly different conduction velocities, producing a compound action potential which underestimated the relative number of slowly conducting fibres.

With increasing time following axotomy there was a progressive decrease in the conduction velocities of all nerve fibres (fig 2). The decline was quantified for fast conducting fibres and slowly conducting fibres respectively by determining the conduction velocities below which $80 \%$ and $20 \%$ of the total number of fibres were represented in the conduction velocity distribution. An estimate of their initial rates of decay following axotomy was obtained by fitting the data with an exponential decay curve of the form $v=v_{0} e^{-t} / T^{T}$ where $v_{0}$ is the control conduction velocity and $T$ the time constant. Fitting the points with a curve of the form $v=v_{1} e^{-t} / T^{T}+v_{2}$, where $v_{1}+v_{2}$ is the control conduction velocity and $v_{2}$ the asymptotic value, gives an estimate of the endpoint of the decay

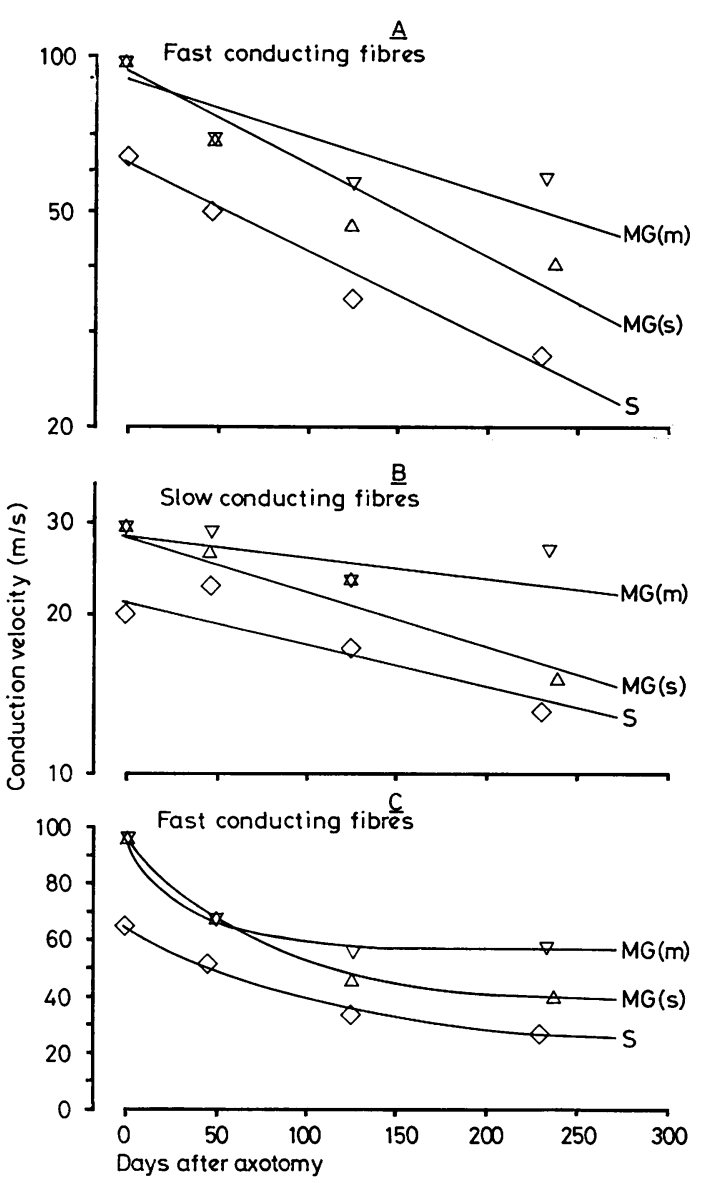

Fig 3 Conduction velocity decay curves. $A$ and $B$ : plots of $v=v_{0} e^{-t} /^{T}$ on a semi-log scale for fast and slowly conducting fibres: sural $(\diamond), M G$ sensory $(\triangle)$ anc! MG motor $(\nabla)$. C: plots of $v=\left.v_{1} e^{-t}\right|^{T}+v_{2}$ on $a$ linear scale for fast conducting fibres. The latter are not shown for slowly conducting fibres, since $v_{2}$ was not significantly different from zero, ie the second equation reduced to the first. Note that the rate of cor:duction velocity decline for fast afferent fibres in $A(S$ and $M G(s))$ is greater than for fast motor fibres $(M G(m))$ or for any of the slowly conducting fibres in $B$. Regression curves were computed from individual data points measured in all 26 experiments, but only averaged data appear in this and subsequent figures (6-8) for clarity. Each symbol represents the mean valice from several experiments, grouped into the time intervals shown in fig 2. Regression parameters are listed in table 1.

prccess (fig 3). Regression curves were calculated using all data points, but for the sake of clarity only the averages of groups of points (grouped into the same time intervals as figs 2,4 , and 5) have 
been plotted in fig 3 . The method of obtaining the regression curves is described in the Appendix.

It was felt that curves of the form $v=v_{0} e^{-t} /^{T}$ gave a better estimate of $\mathrm{T}$ than those of the form $\mathrm{v}=\mathrm{v}_{1} \mathrm{e}^{-\mathrm{t}} / \mathrm{T}+\mathrm{v}_{2}$ since the latter required the introduction of a third parameter $\mathrm{v}_{2}$, which influenced the accuracy with which $v_{1}$ and $T$ could be determined. Furthermore, $v_{2}$ is really the limit of the conduction velocity as $t$ becomes very large. Since the range of observations extended only to 273 days the conduction velocities may have declined further had the nerves remained in their axotomised state for a longer period of time. Values of the regression parameters are listed in table 1 .

The conduction velocity of the fast conducting fibres declined significantly faster than that of the slowly conducting fibres (two-sided $t$-test, $\mathrm{p}<0.0005$ ). However, while the fast conducting fibres had an asymptotic conduction velocity $\mathbf{v}_{2}$, which was significantly greater than zero ( $t$-test, $\mathrm{p}<0.0005$ ), the slowly conducting fibres tended towards a final velocity near zero. However, the computed time constant was so large that it was beyond the period of the experimental observations. Hence, it cannot be concluded that these

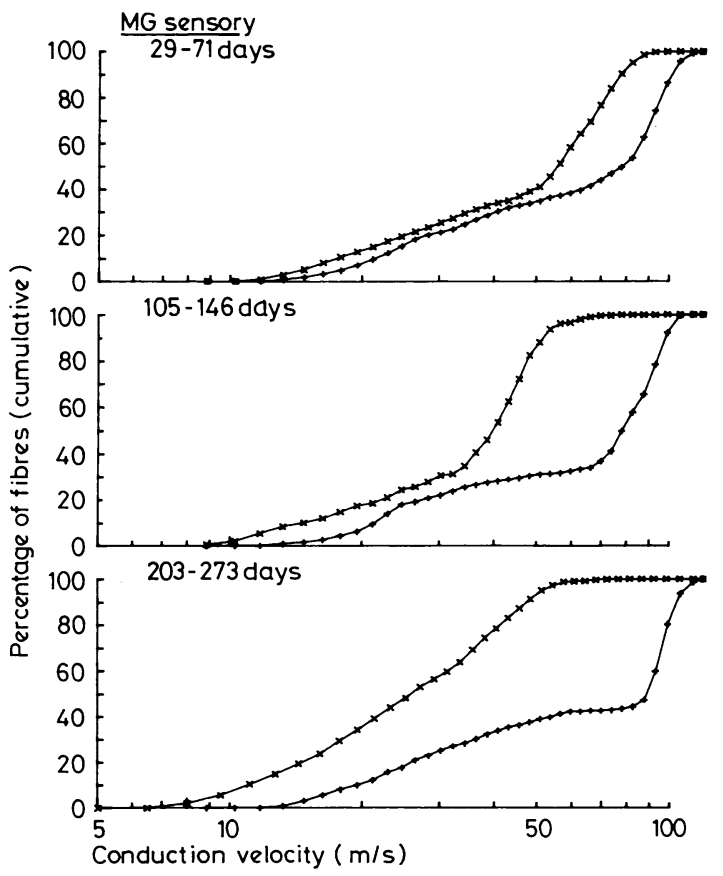

Fig 4 Progressive changes in the conduction velocity distribution of the sensory fibres in $M G$ nerves following axotomy. Conduction velocity distributions are plotted as in fig 2. Refer to text for discussion. fibres actually all died or stopped conducting.

The MG nerve was separated into its sensory and motor components by stimulating either dorsal or ventral roots. The control conduction velocity distribution of the sensory component was also bimodal, although to a lesser extent than the sural nerve. The fast conducting afferent fibres of the MG were approximately $50 \%$ faster than those of the sural. The tendency toward a unimodal conduction velocity distribution following axotomy was much slower in the MG than the sural (fig 4). This may simply reflect a relatively greater difference between the conduction velocities of fast and slow MG afferent fibres, since the corresponding rates of conduction velocity decline did not differ significantly from those of the sural. The same progressive reduction of conduction velocity for all fibres was evident in the MG and the sural nerves. The fast conducting fibres slowed significantly faster than the slowly conducting fibres (two-sided $t$-test, $\mathrm{p}<0.01$ ) but approached an asymptotic value which was significantly greater than zero ( $t$-test, $\mathrm{p}<0.0005$ ). The final value projected for slowly conducting fibres was near zero.

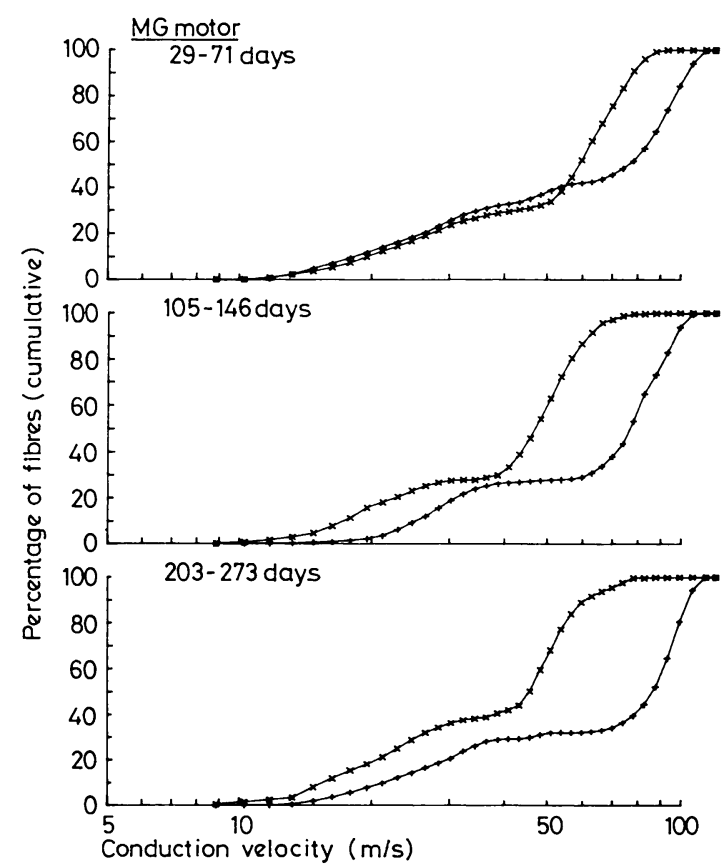

Fig 5 Progressive changes in the conduction velocity distribution of the motor fibres in $M G$ nerves following axotomy. Conduction velocity distributions are plotted as in fig 2. Refer to text for discussion. 
The MG efferent fibres presented a different picture. While the conduction velocity distribution was distinctly bimodal (a clear separation of alphamotoneurones and gamma-motoneurones), the nature of the distribution did not change following axotomy (fig 5). Because the difference in conduction velocities was so great, the fast conducting fibres approached their asymptotic conduction velocity before they reached the slowly conducting fibre range. Although there was no significant difference between the time constants for the conduction velocity decline of fast and slowly conducting fibres, the time constant for slow fibres was more than twice that for fast fibres (table 1). The failure of this difference to be significant is a reflection of the large uncertainty in the value of the time constant for slowly conducting fibres. This uncertainty was partially the product of an EMG artefact which occurred in a number of experiments. This artefact was recorded when stimulating the ventral roots of animals in which all tail and gluteal muscles had not been successfully denervated. In a few experiments the MG nerve was crushed after recording in order to measure the artefact in isolation. It was found to be opposite in sign to the neural potential and to occur with a latency corresponding to that of gamma-motoneurones in control nerves. The artefact was small, but it tended to reduce the amplitude of the later components of the motor compound action potential. Hence, it probably had a more substantial effect on the potentials of axotomised nerves which were smaller and more slowly conducting than those of control nerves.

The asymptotic velocity of the fast conducting efferent fibres was significantly greater than that of the fast conducting afferent fibres in the MG nerve (two-sided $t$-test, $\mathrm{p}<0.0025$ ). This confirmed the earlier finding of Hoffer et $a^{12}$ that the fastest conducting afferent fibres were affected more by axotomy than the fastest efferent fibres. The rate of slowing was also significantly less in efferent fibres (two-sided $t$-test, $\mathrm{p}<0.0025$ ). It therefore appears that degeneration progresses faster and may also continue longer in fast conducting afferent fibres than in alpha-motoneurones.

Hecause of the degree of variability in the conduction velocities for the $20 \%$ level of efferent fibres, it is difficult to infer whether gammamotoneurones were affected to a lesser extent than alpha-motoneurones or slowly conducting afferent fibres.

As noted by Hoffer et al, ${ }^{12}$ the integrated area uncler a compound action potential (referred to as charge because of its dimensions) was dramatically reduced following axotomy. However, it proved to be less straightforward to quantify compound action potential charge decay than conduction velocity decline. There was inevitably some progressive deterioration of compound action potentials during recording which could produce a reduction in amplitude without significantly altering the shape. Consequently, it was possible to extract information about changes in the relative distribution of conduction velocities (determined by the shape of the compound action potential) more reliably than changes in the absolute number of conducting fibres (determined by the magnitude of the compound action potential). Moreover, the absolute number of axons and hence the magnitude of the compound action potential varied from preparation to preparation.

A.nalysis similar to that for conduction velocity was carried out for compound action potential charges recorded both from the spinal roots and the peripheral nerves. Charge was plotted against time after axotomy and the values were fitted with curves of the form $Q=Q_{0} e^{-t} / T$ and

Table 1 Regression parameters for conduction velocity decay curves

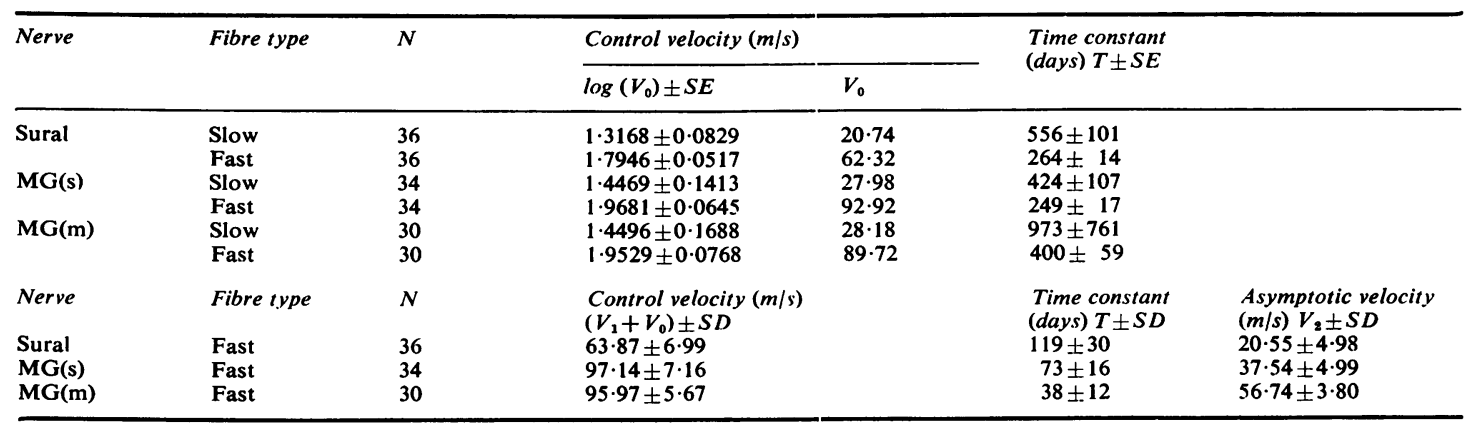

$\mathrm{N}=$ number of cases, $\mathrm{SE}=$ standard error, $\mathrm{SD}=$ standard deviation, $\mathrm{s}=$ sensory $\mathrm{m}=$ motor. 
$\mathrm{Q}=\mathrm{Q}_{1} \mathrm{e}^{-\mathrm{t}} / \mathrm{T}+\mathrm{Q}_{2}$, where $\mathrm{Q}_{0}$ and $\mathrm{Q}_{1}+\mathrm{Q}_{2}$ are the control charge values and $Q_{2}$ is the asymptotic charge value (table 2).

Comparison of the MG sensory and motor compound action potentials, recorded from the dorsal and ventral roots, indicated a significant difference between the rates of decay (two-sided $t$-test, $\mathrm{p}<0.005$ ), the sensory compound action potential charge declining faster than that of the motor compound action potential (results for root charges were combined with the results obtained by Hoffer $e t a l^{12}$ in fig 6). Surprisingly though, there was no significant difference between the rates of decay as determined from nerve compound action potentials. From the relative changes in afferent and efferent conduction velocities, a slower rate of charge decay would have been expected for the motor compound action potentials.

The EMG artefact may have reduced the motor compound action potential charge by a significant amount, affecting axotomised nerves more than control nerves. Since the asymptotic compound action potential charge was less than $40 \%$ of the control value, an EMG artifact which reduced the charge on the control nerve by $10 \%$, for example, could have reduced that of the axotomised nerve by $25 \%$.

The rate of charge decline of sural compound action potentials did not differ significantly from the corresponding rates of either MG sensory or motor compound action potential charges, whether recorded from the spinal roots (fig 6) or the nerve (fig 7). Although not significantly different, the rate of sural charge decay was slower than that of the MG sensory charge. This is probably a consequence of there being a relatively larger number of slowly conducting fibres contributing to the sural compound action potential, along with the fact that the conduction velocity difference between the fastest and slowest conducting fibres is not as great as in the MG. The decline of sural compound action potential charge, therefore, reflects the rate of fast conducting fibre slowing to a lesser extent than does the MG sensory charge.

Conduction velocity distribution values were normalised, scaled by the nth power of the conduction velocity (where $\mathrm{n}$ is the slope obtained from the relationship between the integrated area and conduction velocity of single unit action potentials $^{13}$ and summed in order to obtain the compound action potential charge value expected from a particular conduction velocity distribution. The actual charge decline as recorded from axotomised nerves could then be compared with the expected decline resulting from changes in the conduction velocity distribution alone, making the assumption that the total number of conducting fibres had remained unchanged. A significant difference between the rate constants of recorded and computed charge decay would indicate that other factors had contributed as well.

There was a difference between these two rate constants in all cases (fig 7), the recorded charge

Table 2 Regression parameters for compound action potential charge decay curves

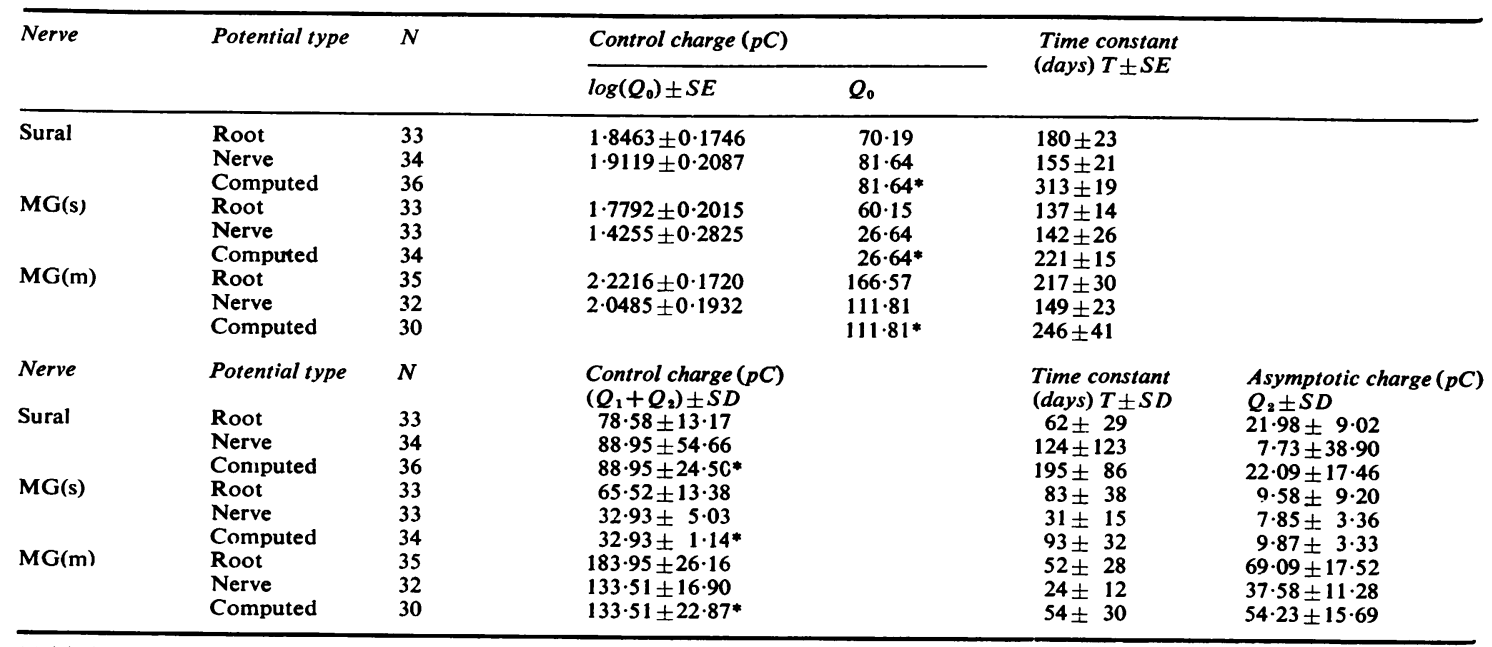

*Initialised to control nerve values.

$\mathrm{N}=$ number of cases, $\mathrm{SE}=$ standard error, $\mathrm{SD}=$ standard deviation, $\mathrm{s}=$ sensory, $\mathrm{m}=\mathrm{motor}$. 


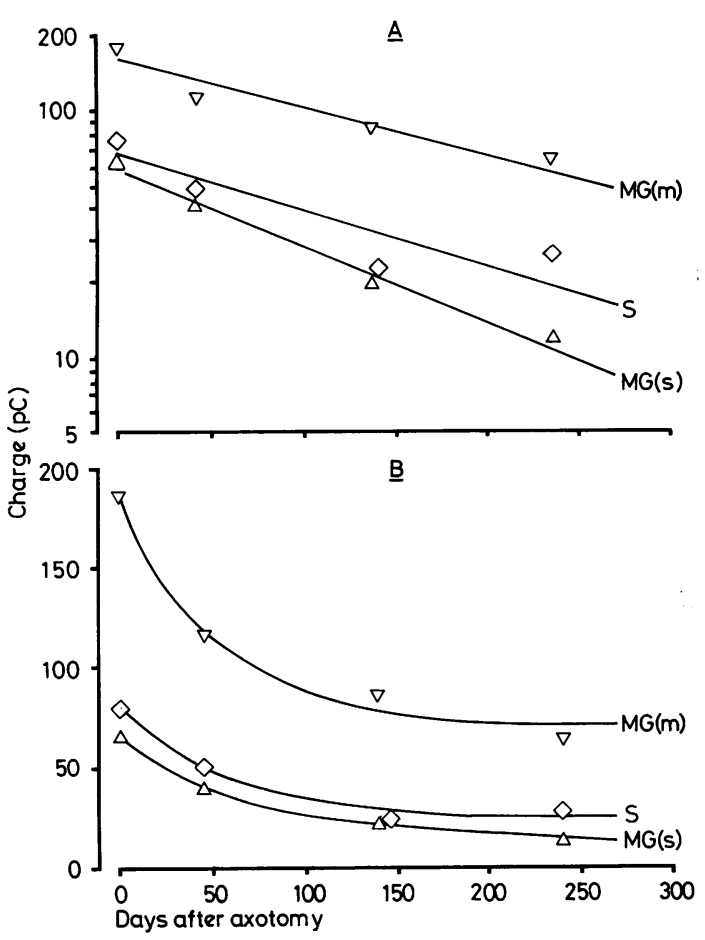

Fig 6 Charge decay curves for compound action potentials recorded from the spinal roots. A: plots of $Q=Q_{0} e^{-t} I^{T}$ on a semi-log scale for compound action potentials recorded from $L 7$ and $S 1$ dorsal roots while stimulating the sural $(\diamond)$ or $M G(\Delta)$ nerves and from the ventral roots while stimulating the $M G$ nerve $(\nabla)$. Each symbol represents the mean from several experiments. Note that the decay of $M G$ sensory charge is faster than MG motor charge. $B$ plots of $Q=Q_{1} e^{-t} T^{T}+Q_{2}$ on a linear scale. Note that $M G$ motor charge approaches a higher level relative to its control level than MG sensory charge. Regression parameters are listed in table 2.

decaying at a faster rate than the computed charge. Except for the MG sensory compound action potential charge, the difference was significant (two-sided $t$-test, $\mathrm{p}<0.0005$ (sural), $\mathrm{p}<0.025$ (MG motor), $0.025<\mathrm{p}<0.05$ (MG sensory)). It would have been significant there as well, had there been slightly less variability in the recorded compound action potential charge.

The discrepancy between the two rate constants must be interpreted as a loss in conducting fibres following axotomy. Fibres may have stopped conducting as the result of degenerative changes following axotomy or they may have stopped conducting as the result of trauma suffered during preparation for recording. Changes definitely did

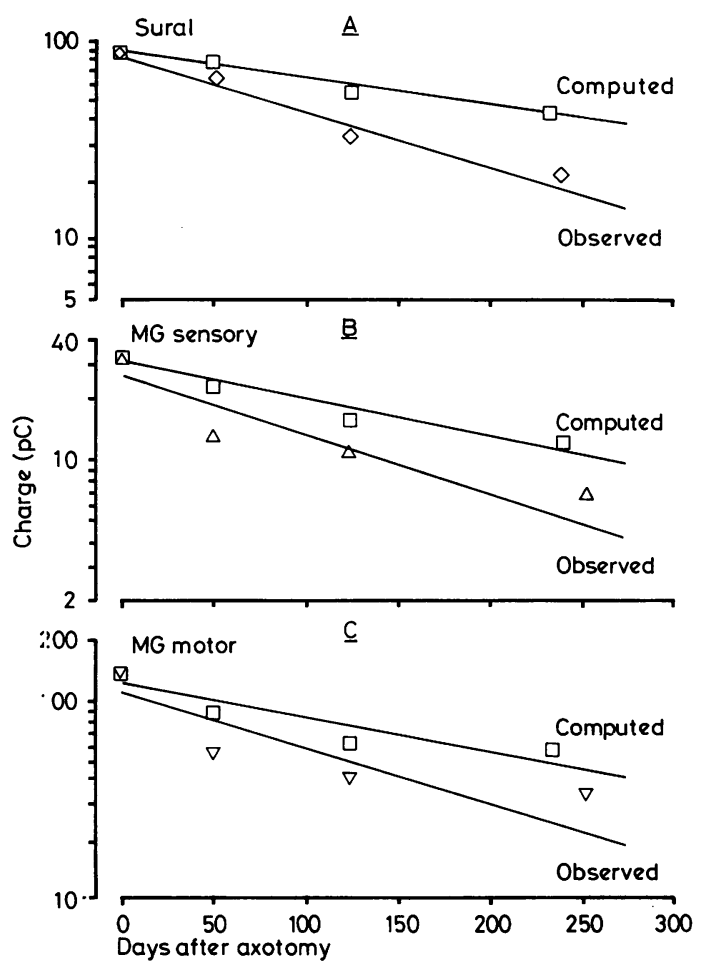

Fig 7 Decay curves of the form $Q=Q_{0} e^{-t} /^{T}$ for compound action potential charge recorded from the ner,es. Observed values are compared with the values exp.ected on the basis of changes in the computed conduction velocity distributions following axotomy. $A:$ : ural: observed charge $(\diamond)$ and computed charge ( $\square)$. B: MG sensory: observed charge $(\triangle)$ and com:puted charge ( $\square)$. C: MG motor: observed chaige $(\nabla)$ and computed charge $(\square)$. Points are plotted as in fig $6 \mathrm{~A}$. Note that the difference between the slopes of recorded and expected charge is similar for all cases. Regression parameters are listed in table 2.

occur while recording from the nerves since compound action potential charge was sometimes reduced by as much as $20-30 \%$ over periods of 15 30 minutes. This was presumably due to loss of axoplasm from the cut nerves combined with concentration changes in the intracellular space and the restricted extracellular space formed by brirging the nerves into paraffin oil for recording. The nerves were ligated to minimise these changes and the compound action potentials generally stabilised, remaining relatively constant for hours afterwards.

The decline of charge during the course of recording compound action potentials occurred in 
both control and axotomised nerves to more or less the same degree. Therefore, the rate constants of the exponential charge decay should not have been altered significantly. Based on this assumption, it must be concluded that a significant number of fibres stopped conducting in response to axotomy, although the fact that the recorded nerve charge values appear to reach non-zero limits (fig 8) implies that a certain population of fibres retain the ability to conduct action potentials, perhaps indefinitely.

Calculations of the total number of fibres in the conduction velocity distributions support this conclusion. Nerves were separated into three groups: control, 0-105 days after axotomy and 126-273 days after axotomy. The mean number of fibres

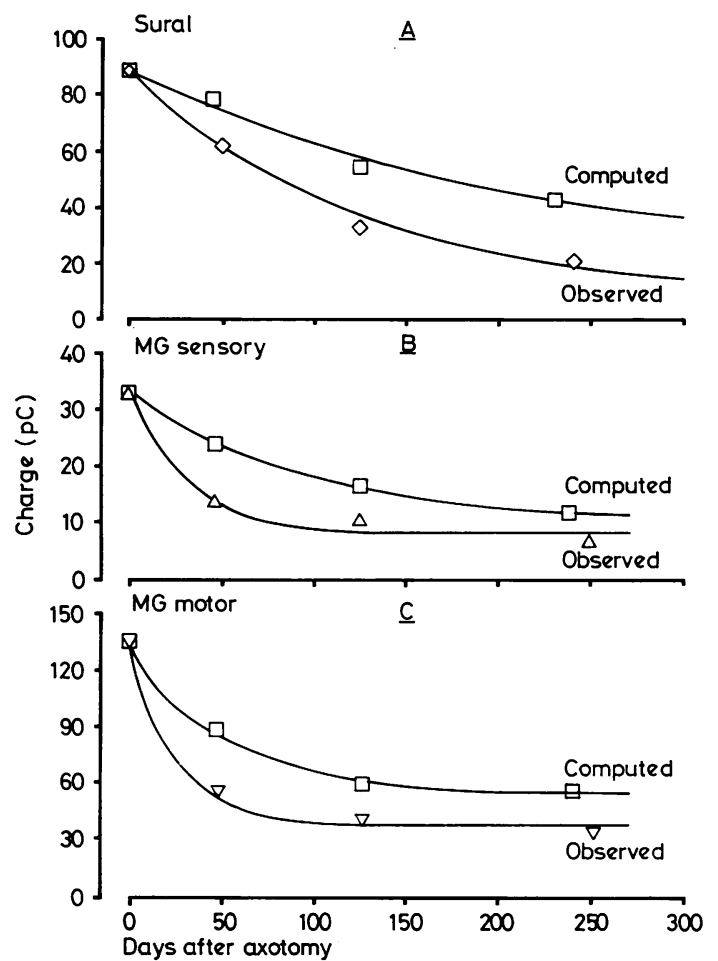

Fig 8 Decay curves of the form $Q=Q_{1} e^{-t} /^{T}+Q_{2}$ for compound action potential charge recorded from the nerves. Observed values are compared with the values expected on the basis of changes in the computed conduction velocity distributions following axotomy. A: sural: observed charge $(\diamond)$ and computed charge ( $\square)$. B: $M G$ sensory: observed charge $(\triangle)$ and computed charge ( $\square$ ). C: MG motor: observed charge $(\nabla)$ and computed charge $(\square)$. Points are plotted as in fig $6 B$. Regression parameters are listed in table 2.
Table 3 Computed number of conducting nerve fibres

\begin{tabular}{|c|c|c|c|c|c|c|}
\hline \multirow[t]{2}{*}{ Nerve } & \multicolumn{2}{|l|}{ Control } & \multicolumn{2}{|l|}{ 29-105 Days } & \multicolumn{2}{|c|}{ 126-273 Davs } \\
\hline & $M e a n \pm S E$ & $N$ & $M e a n \pm S E$ & $N$ & $M e a n \pm S E$ & $N$ \\
\hline $\begin{array}{l}\text { Sural } \\
\text { MG (s) } \\
\text { MG (m) }\end{array}$ & $\begin{array}{r}1135 \pm 104 \\
277 \pm 24 \\
745 \pm 84\end{array}$ & $\begin{array}{l}\text { (12) } \\
\text { (14) } \\
\text { (14) }\end{array}$ & $\begin{array}{l}847 \pm 99 \\
164 \pm 37 \\
520 \pm 107\end{array}$ & $\begin{array}{l}\text { (8) } \\
\text { (8) } \\
\text { (8) }\end{array}$ & $\begin{array}{l}365 \pm 77 \\
156 \pm 40 \\
406 \pm 90\end{array}$ & $\begin{array}{l}\text { (6) } \\
\text { (6) } \\
\text { (5) }\end{array}$ \\
\hline
\end{tabular}

$\mathrm{N}=$ number of cases, $\mathrm{SE}=$ standard error, $\mathrm{s}=$ sensory, $\mathrm{m}=$ motor.

computed for each group is listed in table 3. There was no significant difference between the control mean and the 29-105 day mean for either the sural (two-sided $t$-test, $0.025<\mathrm{p}<0.05$ ) or the MG motor fibres (two-sided $t$-test, $p>0.05$ ). Only the $M G$ sensory fibres showed a significant difference (twosided $t$-test, $\mathrm{p}<0.01$ ), but the 126-273 day mean was significantly less than the control in all cases (two-sided $t$-test, $\mathrm{p}<0.0005$ (sural), $\mathrm{p}<0.025$ (MG motor), $\mathrm{p}<0.01$ (MG sensory)).

\section{Discussion}

The results of the present series of experiments indicate that, while changes in conduction velocity distributions account for a large proportion of the loss of compound action potential charge following axotomy, there is a significant loss in the number of conducting fibres. Fast conducting efferent fibres degenerate less rapidly than either fast conducting muscle or cutaneous afferent fibres. In contrast, there is little difference between the rates of degeneration of slowly conducting fibres in any of these three categories. Furthermore, fast conducting afferent fibres of both types degenerate faster than slowly conducting afferents.

The present study not only confirms the finding of Hoffer et al.12 "that following axotomy large myelinated sensory fibres are substantially more affected than motor fibres in the same peripheral nerves," but also shows that muscle and cutaneous afferent fibres are affected to more or less the same degree. The observed declines in conduction velocity and compound action potential charge are to a large extent the products of nerve fibre atrophy. As the total fibre diameter decreases, conduction velocity slows with a concomitant reduction in the amplitude and charge of a single unit potential. This is well-known from empirical data $^{15}$ and is predicted on the basis of theoretical considerations.1617 Fibre diameter distributions determined from histological sections, however, do not appear to be good measures of the conduction velocity distributions of degenerating nerves. Cumulative fibre diameter histograms, derived from measurements of total fibre diameter, showed 
a much less pronounced shift toward the smaller end of the spectrum than the corresponding shift toward slower conduction velocities in the conduction velocity distributions (Gillespie and Hanley, unpublished observations).

Arbuthnott et al $^{18}$ made a careful comparison of conduction velocity and ultrastructural characteristics of nerve fibres and found that conduction velocity varied quite linearly with axon circumference over the entire spectrum of cat sensory and motor nerve fibres. Since axon diameter tends to be reduced relatively more than myelin following axotomy (references can be found in Sunderland ${ }^{1}$ ), the reduction of the total diameter would be an underestimate of the reduction in axon circumference. Hence, changes in total fibre diameter (axon +myelin) could underestimate the changes in conduction velocity.

Cragg and Thomas ${ }^{3}$ compared changes in conduction velocity with changes in axon diameter of the largest fibres in atrophying nerves. In reanalysing the data in their fig 5B it appears that for changes in conduction velocity between $0 \%$ and $-20 \%$ there is a relationship which is approximately linear (correlation coefficient $=0 \cdot 84$; slope $=$ 0.86). However, in the same figure there are fibres whose conduction velocity changed by up to $-45 \%$ with little further change in axon diameter. Thus, an additional mechanism must be operating in these extreme cases, for example a pulling away of myelin from the internodal region. A short region of demyelination can produce a substantial slowing of conduction or even block conduction completely. ${ }^{19-21}$ These mechanisms can now be examined experimentally, so there is promise that the interaction between axons and their myelin sheaths following a distal nerve lesion can be understood in some detail.

Hoffer et $a l^{12}$ were unable to determine the extent of cell death, if any, following axotomy. Carlson $e t a l^{22}$ claimed that there was no significant loss in the number of motor axons or cell bodies 18 months after amputation of the hindlimb, although nerve fibres in the L7 ventral ruot were reduced in diameter. In contrast, there was a significant loss of both dorsal root fibres and ganglion cells (approximately $20 \%$ ) in addition to a reduction in fibre diameter. Although such a loss of dorsal root ganglion cells might account for some of the difference between charge computed from the sensory conduction velocity distribution and the actual recorded charge, it seems unlikely that it could explain the discrepancy completely since they saw the greatest loss among the smaller cell bodies. Furthermore, their findings lead one to expect that the charge recorded from efferent fibres should not decay significantly faster than that computed from the conduction velocity distribution. Since they do not describe the appearance of any of the surviving fibres, no assessment can be made of the ability of these fibres to conduct impulses.

Recently, Jessell et $a l^{23}$ have shown that there is a $75-80 \%$ depletion of substance $P$ from the dorsal horn following sciatic nerve section. They suggest that this probably reflects the degeneration of substance $\mathbf{P}$ containing neurones. This is consistent with Carlson's findings mentioned above, sirce so far substance $P$ has been identified only in the smaller diameter myelinated afferents. ${ }^{24}$ Thus, although much of the decay in compound action potential charge is due to slowing of conduction in atrophying fibres, the percentage which results from failure of conduction or cell death or both has still not been clearly resolved.

Support for the differential degeneration of fast and slow conducting afferent fibres may be derived from differences in the rates of regeneration and maturation following nerve crush. Devor and Govrin-Lippmann ${ }^{25}$ have shown that fast conducting fibres regenerate more quickly than slowly conducting fibres and that recovery of conduction velocity in regenerating sprouts occurs at faster rates in fast conducting fibres than slowly conducting fibres. ${ }^{20}$

The present study offers no way of distinguishing between the roles played by trophic factors and ongoing electrical activity in maintaining the viatbility of nerve fibres. Czéh et $a l^{27}$ have shown that while disuse of muscle afferents does cause a small reduction in conduction velocity, this slcwing is substantially less than that observed following axotomy. Similar disuse of alphamotoneurones ${ }^{28}$ leads only to changes in the electrical properties of the cell body without affecting axonal conduction velocity. These findings suggest that trophic factors play a primary role in preserving the functionality of an axon.

We feel, however, that electrical activity may be significant in determining the differential effects of axotomy on various classes of axons. Fast conducting afferents should experience the greatest reduction in impulse traffic following axotomy, relative to that present in normal fibres. Alphamotoneurones and presumably gamma-motoneurones continue to be excited centrally, ${ }^{29}$ while small afferents terminate in relatively unspecialised endings and continue to be excited by pressure or temperature changes in a neuroma. ${ }^{30}$ Therefore, the most dramatic effects should occur in the large 
afferent group of fibres, which only remain active for a short period after ligation. ${ }^{6}$ The exact nature of any trophic role played by electrical activity is not yet known though and awaits further investigation.

\section{Appendix}

Data points were fitted with curves of the form $\mathrm{y}=\mathrm{Ae}^{-\mathrm{Bt}}$ or $\mathrm{y}=\mathrm{kv}^{\mathrm{n}}$ using the transformations $(\ln (\mathrm{y})$ $=\ln (\mathrm{A})-\mathrm{Bt}$ or $\ln (\mathrm{y})=\ln (\mathrm{k})+\mathrm{n} \ln (\mathrm{v})$ respectively. Correlation coefficients and standard errors were calculated according to standard equations for linear regression. ${ }^{31}$ Regression coefficients from different populations were tested for significant differences by testing the null hypothesis. Decay curves of the form $\mathrm{Ae}^{-\mathrm{Bt}}+\mathrm{C}$ were obtained by computing parameters which minimised the residual sum of squares. A nonlinear regression analysis programme was employed. This programme is available in the BMDP package of programmes for biomedical applications developed at the Health Sciences Facility of the University of California, Los Angeles.

This research was supported by grants from the Canadian Medical Research Council and the Muscular Dystrophy Association of Canada to RBS and by a Canadian Medical Research Council studentship to TEM.

\section{References}

1 Sunderland S. Nerve and Nerve Injuries. Edinburgh: Livingstone, 1978.

2 Kiraly JK, Krnjević K. Some retrograde changes in function of nerves after peripheral section. $Q$ J Exp Physiol 1959; 44:244-57.

3 Cragg BG, Thomas PK. Changes in conduction velocity and fibre size proximal to peripheral nerve lesions. J Physiol (Lond) 1961; 157:315-27.

4 Gutmann E, Sanders FK. Recovery of fibre numbers and diameters in the regeneration of peripheral nerves. J Physiol (Lond) 1943; 101:489518.

5 Cragg BG, Thomas PK. The conduction velocity of regenerated peripheral nerve fibres. $J$ Physiol (Lond) 1964; 171:164-75.

6 Govrin-Lippmann R, Devor M. Ongoing activity in severed nerves: source and variation with time. Brain Res 1978; 159:406-10.

7 Davis LA, Gordon T, Hoffer JA, Jhamandas J, Stein RB. Compound action potentials recorded from mammalian peripheral nerves following ligation or resuturing. J Physiol (Lond) 1978; 285: 543-59.

8 Stein RB, Hoffer JA, Gordon T, Davis LA, Charles D. Long-term recordings from cat peripheral nerves during degeneration and regeneration: implications for human nerve repair and prosthetics. In: Jewett DL and McCarrol HR, eds. Nerve Repair: Its Clinical and Experimental Basis. St Louis: CV Mosby, 1979; 166-76.

9 Fleming RA. Some notes on ascending degeneration (so-called) and on the changes in nerve cells consequent thereon. Br Med J 1896; 2:918-21.

10 Bucy PC. Studies in degeneration of peripheral nerves. J Comp Neurol 1928; 45:129-59.

11 Gutmann E, and Holubář J. The degeneration of peripheral nerve fibres. J Neurol Neurosurg Psychiatry 1950; 13:89-105.

12 Hoffer JA, Stein RB, Gordon T. Differential atrophy of sensory and motor fibers following section of cat peripheral nerves. Brain Res 1979; 178:347-61.

13 Milner TE, Stein RB, Gillespie J, Hanley B. Improved estimates of conduction velocity distributions using single unit action potentials. $J$ Neurol Neurosurg Psychiatry 1981; 44:476-84.

14 Blair EA Erlanger J. A comparison of the characteristics of axons through their individual electrical responses. Am J Physiol 1933; 106:524-64.

15 Gasser HS, Grundfest H. Axon diameters in relation to the spike dimensions and the conduction velocity in mammalian A fibres. $A m$ J Physiol 1939; 127:393-414.

16 Stein RB, Pearson KG. Predicted amplitude and form of action potentials from unmyelinated nerve fibres. J Theor Biol 1971; 32:539-58.

17 Moore JW, Joiner RW, Brill MH, Waxman SG, Najaar-Joa M. Simulations of conduction in uniform myelinated fibers. Relative sensitivity to changes in nodal and internodal parameters. Biophys J 1978; 21:147-60.

18 Arbuthnott ER, Boyd IA, Kalu KU. Ultrastructural dimensions of myelinated peripheral nerve fibres in the cat and their relation to conduction velocity. J Physiol (Lond) 1980; 308: 125-58.

19 Koles ZJ, Rasminsky M. A computer simulation of conduction in demyelinated nerve fibres. $J$ Physiol (Lond) 1972; 227:351-64.

20 Bostock H, Sears TA. The internodal axon membrane: electrical excitability and continuous conduction in segmental demyelination. J Physiol (Lond) 1978; 280:273-301.

21 Waxman SG. Prerequisites for conduction in demyelinated fibers. Neurology (Minneap) 1978; 28(2): 27-33.

22 Carlson J Lais A, Dyck PJ. Axonal atrophy from permanent peripheral axotomy in adult cats. $J$ Neuropathol Exp Neurol 1979; 38:579-85.

23 Jessell T, Tsunoo A, Kanazawa I, Otsuka M. Substance P: depletion in the dorsal horn of rat spinal cord after section of the peripheral processes of primary sensory neurons. Brain Res 1979; 168:247-59.

24 Hökfelt T, Johansson O, Kellerth J-O et al. Immunohistochemical distribution of substance $P$. 
In: von Euler US and Pernow B, eds. Substance $P$. New York: Raven Press, 1977; 117-45.

25 Devor M, Govrin-Lippmann R. Maturation of axonal sprouts after nerve crush. Exp Neurol 1979; 64:260-70.

26 Devor M, Govrin-Lippmann R. Selective regeneration of sensory fibers following nerve crush injury. Exp Neurol 1979; 65:243-54.

27 Czéh G, Kudo N, Kuno M. Membrane properties and conduction velocity in sensory neurones following central or peripheral axotomy. J Physiol (Lond) 1977; 270:165-80.

28 Czéh G, Gallego R, Kudo N, Kuno M. Evidence for the maintenance of motoneurone properties by muscle activity. J Physiol (Lond) 1978; 281: 239-52.

29 Gordon T, Hoffer JA, Jhamandas J, Stein RB. Long-term effects of axotomy on neural activity during cat locomotion. J Physiol (Lond) 1980; 303:243-63.

30 Wall PD, Gutnick M. Ongoing activity in peripheral nerves: the physiology and pharmacology of impulses originating from a neuroma. Exp Neurol 1974; 43:580-93.

31 Edwards AL. An Introduction to Linear Regression and Correlation. San Francisco: WH Freeman, 1976. 